\title{
Power-law distributions for the areas of the basins of attraction on a potential energy landscape
}

\author{
Claire P. Massen \\ Department of Chemistry, University of Cambridge, Lensfield Road, Cambridge CB2 1EW, United Kingdom \\ Jonathan P. K. Doye* \\ Physical and Theoretical Chemistry Laboratory, Oxford University, South Parks Road, Oxford OX1 3QZ, United Kingdom
}

(Received 7 September 2005; revised manuscript received 8 December 2006; published 19 March 2007)

Energy landscape approaches have become increasingly popular for analyzing a wide variety of chemical physics phenomena. Basic to many of these applications has been the inherent structure mapping, which divides up the potential energy landscape into basins of attraction surrounding the minima. Here, we probe the nature of this division by introducing a method to compute the basin area distribution and applying it to some archetypal supercooled liquids. We find that this probability distribution is a power law over a large number of decades with the lower-energy minima having larger basins of attraction. Interestingly, the exponent for this power law is approximately the same as that for a high-dimensional Apollonian packing, providing further support for the suggestion that there is a strong analogy between the way the energy landscape is divided into basins, and the way that space is packed in self-similar, space-filling hypersphere packings, such as the Apollonian packing. These results suggest that the basins of attraction provide a fractal-like tiling of the energy landscape, and that a scale-free pattern of connections between the minima is a general property of energy landscapes.

DOI: 10.1103/PhysRevE.75.037101

The potential energy surface, which defines how the potential energy depends on the coordinates of all the atoms in a system, has a complex, multidimensional landscape [1]. In recent years, there have been intensive efforts to understand the behavior of systems, such as proteins, supercooled liquids and clusters, in terms of features of these energy landscapes [2]. This research programe has led to important insights into how proteins fold [3] and the origins of the unusual dynamic properties of supercooled liquids $[4,5]$.

Many of these applications rely on the inherent structure mapping introduced by Stillinger and Weber [6] that is illustrated in Fig. 1. It divides the energy landscape into basins of attraction surrounding the minima on the energy landscape, where a basin is defined as the set of points for which following the steepest-descent paths downhill from those points leads to the same minimum. The utility of the inherent structure approach is that it allows the behavior of the complete landscape to be conceived in terms of the properties of the individual basins [7], which are themselves tractable to calculate. Hence, landscape-based descriptions of a system's thermodynamics and dynamics can be obtained [1].

In many of the analyses of energy landscapes, the aim has been to understand differences in behavior, e.g., proteins that are good or bad folders [8,9], or supercooled liquids that show strong or fragile dynamics $[10,11]$, in terms of differences in the energy landscape. However, there has been much less attention on the more universal organizing principles that are common to all such complex landscapes. For example, it is known that the number of minima scales exponentially with system size [12], and the distribution of minima as a function of energy is a Gaussian [13], but what is the nature of the division of the landscape into basins and

\footnotetext{
*Electronic address: jonathan.doye@chem.ox.ac.uk
}

the pattern of connectivities between these basins? In particular, does the inherent structure mapping lead to an equitable division of configuration space into basins, or an inequitable one where a small minority of the basins occupy a substantial majority of the space?

Clues from recent work on the energy landscapes of small Lennard-Jones clusters $[14,15]$ perhaps suggest the latter scenario. The network of minima connected by transition states was found to be scale-free [16], that is the probability distribution for the degree (the number of connections to a node in the network) has a power-law tail. Such a feature has been found for many technological, social, and biochemical networks $[17,18]$, however, unlike these other networks where the scale-free behavior arises from the dynamics of network growth [16], these inherent structure networks are static. Hence the origin of the scale-free topology remains a puzzle.

One potential answer is that the network structure reflects

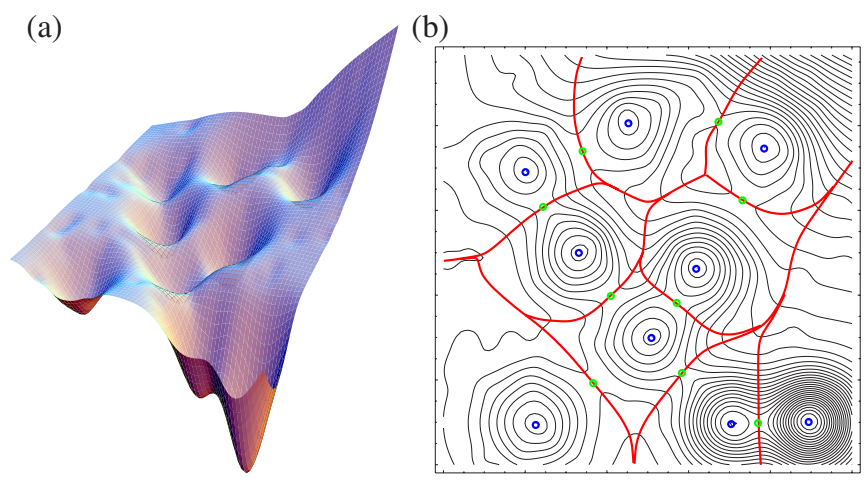

FIG. 1. (Color online) (a) A model two-dimensional energy landscape and (b) its associated contour plot illustrating the inherent structure mapping. In (b) the landscape has been divided into basins of attraction where the basin boundaries are represented by the thick lines, and the minima and transition states by points. 


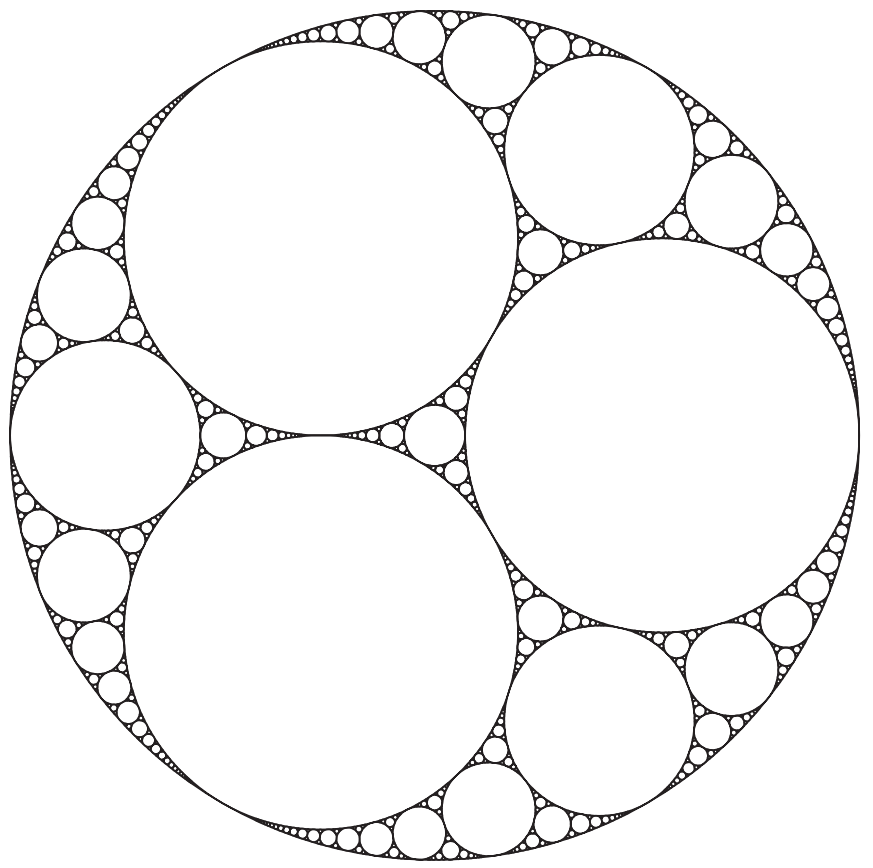

FIG. 2. An Apollonian packing of a circle. Such a space-filling packing of disks is obtained iteratively, starting from an initial configuration of three mutually touching disks inside the circle. At each iteration disks are added inside each empty curvilinear triangle, such that each disk touches all three disks bounding the triangle. This process is repeated ad infinitum creating a space-filling fractal packing of the circle. Its fractal dimension is 1.3057 [25].

an inequitable division of configuration space with the lowenergy, high-degree minima having the largest basins of attraction $[19,20]$, because they are then more likely to have more transition states along the long basin boundaries. Indeed, a recently proposed model spatial, scale-free network based upon Apollonian packings [21] has just such a correlation between area and degree [22,23]. A two-dimensional example of an Apollonian packing is illustrated in Fig. 2. Such a packing is generated by the iterative addition of eversmaller disks into the interstices of the packing until the space is filled. Consequently, the packing is fractal and selfsimilar [24]. Importantly, the network of contacts between the disks is scale-free, and the properties of these Apollonian networks are very similar to the inherent structure networks $[22,23]$. Thus, there seems to be a potential analogy between the hyperspheres in a high-dimensional Apollonian packing and the basins of attraction on an energy landscape. However, the starting point for this argument was the energy landscapes for clusters with less than 15 atoms, and it is not clear whether the energy landscapes for these very small systems are representative.

The fractal nature of the Apollonian packings is evident from the distribution of the radii of the disks or hyperspheres making up the packing. This distribution is a power law at small radii with the exponent related to the fractal dimension, $d_{f}$, where $d-1<d_{f}<d$ and $d$ is the dimension of the space being packed [26]. This feature potentially allows the analogy between the Apollonian packings and the energy landscapes to be tested further. The distribution of the vol- umes of the hyperspheres in an Apollonian packing scales as $V^{-\left(1+d_{f} / d\right)}$, thus leading to the prediction that, because of the high dimensionality of typical configuration spaces, the distribution of the hyperareas of the basins of attraction will follow a power law with exponent -2 , if the basins tile configuration space in an Apollonian-like manner.

To test this prediction we need a method that can obtain this basin area distribution. To achieve this we study a transformed potential energy surface that is commonly used in global optimization and is particularly associated with the "basin-hopping" approach [27,28]. This transformation involves assigning the energy at a particular point in configuration space to that obtained after performing a local minimization from that point. It transforms the landscape into a set of steps, where each corresponds to a basin of attraction surrounding a minimum on the original potential energy surface. Thus, for the example landscape in Fig. 1, the transformed landscape would consist of nine steps each having the energy of the corresponding minimum. Importantly, the probability of sampling a step during a simulation on the transformed landscape is proportional to its hyperarea $[19,29]$.

Specifically, if we assume that the properties of the basins are uniquely characterized by the energy of their minima, the probability of being on a step with potential energy in the range $E \pm d E / 2$ in the canonical ensemble obeys

$$
p_{\text {min }}^{\text {trans }}(E, T) d E \propto \Omega_{\text {min }}(E) A(E) \exp (-E / k T) d E,
$$

where $\Omega_{\min }(E)$ is the number of minima with energy $E \pm d E / 2$ and $A(E)$ is the average area of the basins of attraction surrounding minima with energy $E$. Hence, we can obtain both $A(E)$ (to within a multiplicative constant) and the basin area distribution from $p_{\min }^{\text {trans }}(E, T)$ distributions obtained from simulations, if we first know $\Omega_{\text {min }}(E)$. Methods to obtain $\Omega_{\min }(E)$ have already been developed $[13,30]$, and involve inverting similar probability distributions for the original potential energy landscape. Namely, the probability of being in the basin of attraction of a minimum with potential energy in the range $E \pm d E / 2$ in the canonical ensemble is given by

$$
p_{\min }(E, T) d E \propto \Omega_{\min }(E) Z_{\mathrm{vib}}(E, T) \exp (-E / k T) d E,
$$

where $Z_{\mathrm{vib}}(E, T)$ is the vibrational partition function of minima with energy $E$, which can be calculated, for example, using the harmonic approximation with frequencies obtained by diagonalization of the Hessian matrix of the relevant minima.

Here, we apply this scheme [31] to three representative systems that have been much studied in the supercooled liquids community [32-34], namely a binary Lennard-Jones mixture with approximate composition $A_{4} B$ and LennardJones parameters as given in Ref. [35]; a model onecomponent glass-forming liquid interacting with the Dzugutov potential [36]; and amorphous silicon modelled by the Stillinger-Weber potential [37] with a strengthened threebody term [34]. All three systems have 256 atoms and are modelled using periodic boundary conditions. 
For each system, we ran two sets of simulations. First, constant-temperature molecular dynamics was performed and by regularly minimizing configurations generated along the trajectory we obtained $p_{\min }(E, T)$ and hence $\Omega_{\min }(E)$ (and a Gaussian fit to it). We chose temperatures well above the glass transition where equilibrium sampling of the liquid is easy to obtain. Second, Metropolis Monte Carlo simulations were performed on the transformed landscape, from which $p_{\min }^{\text {trans }}(E, T)$ and hence $A(E)$ was obtained. As a check of the sampling, $p_{\min }^{\operatorname{trans}}(E, T)$ distributions were collected at several temperatures. The resulting area distributions were in good agreement, confirming the reliability of the approach.

In Fig. 3(a), $A(E)$ is depicted for these three systems. In agreement with our expectation that the deeper, more connected minima should have larger basins of attraction, the basin areas decrease very rapidly with increasing energy in an approximately exponential manner. The probability distributions for the hyperareas of the basins of attraction are illustrated in Fig. 3(b). It is apparent that the distributions follow an approximate power law over the whole range of areas sampled, which is between 13 and 18 decades depending on the system. Closer inspection shows that the distributions begin to curve slightly downwards for larger areas, but that for the smaller basins the distributions very closely follow the prediction from the Apollonian analogy for 8 to 9 decades for the binary Lennard-Jones and Dzugutov systems, and 5 decades for amorphous silicon.

These results have a number of important implications for the fundamental properties of energy landscapes, and the inherent structure mapping in particular. First, they show that this mapping produces a very heterogeneous division of configuration space with the deeper basins having much larger basins of attraction. Second, there appears to be a strong analogy between the way that basins of attraction tile the energy landscape, and hyperspheres fill space in an Apollonian packing. This similarity is exemplified by the power-law distribution of the basin areas at small $A$, which has exactly the expected exponent over a very wide range of basin areas, and suggests that the basins divide configuration space in a fractal-like manner. Of course, the analogy must break down at some sufficiently small length scale, because the number of minima on an energy landscape, although large, is necessarily finite, whereas the Apollonian packings require an infinite number of hyperspheres to fill space. However, there is not yet any sign of this breakdown in the energy range that we have sampled. We should note that this energy range corresponds to the parts of the PEL sampled by a liquid, and in future work it would be interesting to try to use enhanced and biased sampling techniques to sample the basin area distribution at both higher and lower energies.

Third, if, as for the Apollonian networks, the degree is a power-law function of the area [23] (or equivalently the hyperlength of the basin boundary) the power-law distribution of basin areas is a signature that the underlying pattern of connections between the minima is scale-free. Thus, our results suggest that this scale-free topology is not just specific to small Lennard-Jones clusters, but a more universal prop-
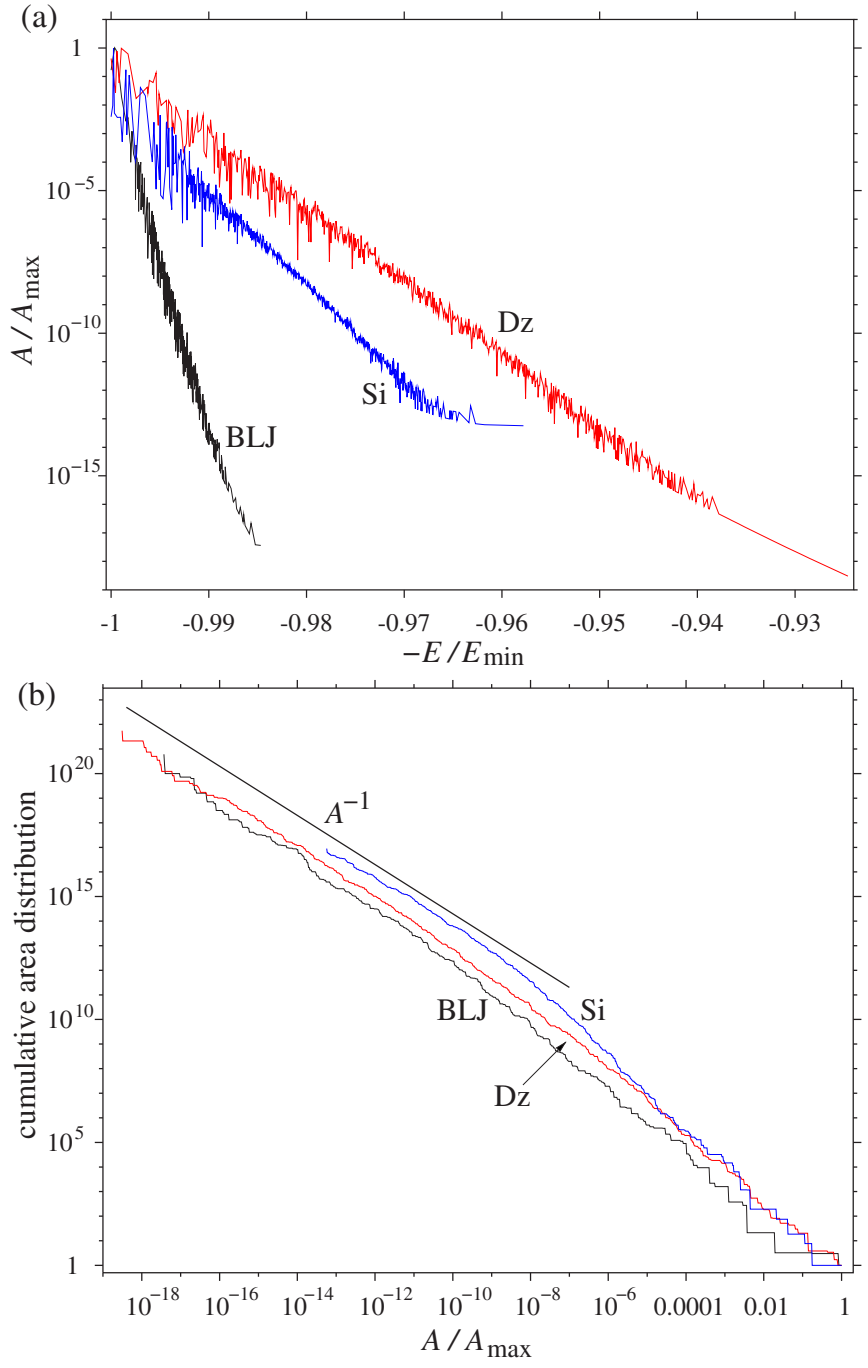

FIG. 3. (Color online) (a) The dependence of the basin area on the energy of the minima, and (b) the cumulative basin area distributions for the binary Lennard-Jones (BLJ) and Dzugutov (Dz) liquids, and amorphous $\mathrm{Si}$. To aid comparison between these systems, the basin area and the energy are measured with respect to the largest basin and the lowest-energy minima that have been sampled, respectively. Additionally, in (b) the power-law predicted by the analogy to Apollonian packings has been added for comparison [38].

erty of energy landscapes. Indeed, there is evidence that the energy landscapes of polypeptides also have a scale-free character [39]. Fourth, the fractal-like character of the energy landscape provides a static explanation of the origin of the scale-free topology of inherent structure networks. However, this is not the end of the matter, since why the basins provide a fractal-like tiling of the energy landscape is still a puzzle, and one which we will explore in future work.

The authors are grateful to the Engineering and Physical Sciences Research Council (C.P.M) and the Royal Society (J.P.K.D) for financial support. 
[1] D. J. Wales, Energy Landscapes (Cambridge University Press, Cambridge, 2003).

[2] C. L. Brooks III, J. N. Onuchic, and D. J. Wales, Science 293, 612 (2001).

[3] C. M. Dobson, A. J. Šali, and M. Karplus, Angew. Chem., Int. Ed. 37, 868 (1998).

[4] F. H. Stillinger, Science 267, 1935 (1995).

[5] P. G. Debenedetti and F. H. Stillinger, Nature (London) 410, 259 (2001).

[6] F. H. Stillinger and T. A. Weber, Science 225, 983 (1984).

[7] F. Sciortino, J. Stat. Mech.: Theory Exp. (2005) P05015.

[8] A. Sali, E. Shakhnovich, and M. Karplus, Nature (London) 369, 248 (1994).

[9] J. D. Bryngelson, J. N. Onuchic, N. D. Socci, and P. G. Wolynes, Proteins 21, 167 (1995).

[10] S. Sastry, Nature (London) 409, 164 (2001).

[11] G. Ruocco, F. Sciortino, F. Zamponi, C. de Michele, and T. Scopigno, J. Chem. Phys. 120, 10666 (2004).

[12] F. H. Stillinger, Phys. Rev. E 59, 48 (1999).

[13] F. Sciortino, W. Kob, and P. Tartaglia, Phys. Rev. Lett. 83, 3214 (1999).

[14] J. P. K. Doye, Phys. Rev. Lett. 88, 238701 (2002).

[15] J. P. K. Doye and C. P. Massen, J. Chem. Phys. 122, 084105 (2005).

[16] A. L. Barabási and R. Albert, Science 286, 509 (1999).

[17] R. Albert and A. L. Barabási, Rev. Mod. Phys. 74, 47 (2002).

[18] M. E. J. Newman, SIAM Rev. 45, 167 (2003).

[19] J. P. K. Doye, D. J. Wales, and M. A. Miller, J. Chem. Phys. 109, 8143 (1998).

[20] T. V. Bogdan, D. J. Wales, and F. Calvo, J. Chem. Phys. 124, 044102 (2006)

[21] J. S. Andrade, H. J. Herrmann, R. F. S. Andrade, and L. R. da Silva, Phys. Rev. Lett. 94, 018702 (2005).

[22] J. P. K. Doye and C. P. Massen, Phys. Rev. E 71, 016128 (2005).

[23] J. P. K. Doye and C. P. Massen, in Complexity, Metastability and Nonextensivity, edited by C. Beck, G. Benedek, A. Rapisarda, and C. Tsallis (World Scientific, Singapore, 2005), pp. 375-384; e-print cond-mat/0612150.

[24] B. B. Mandelbrot, The Fractal Geometry of Nature (W. H. Freeman, New York, 1983).

[25] S. S. Manna and H. J. Herrmann, J. Phys. A 24, L481 (1991).

[26] D. W. Boyd, Mathematika 20, 170 (1973).

[27] D. J. Wales and J. P. K. Doye, J. Phys. Chem. A 101, 5111 (1997).

[28] D. J. Wales and H. A. Scheraga, Science 285, 1368 (1999).

[29] J. P. K. Doye and D. J. Wales, Phys. Rev. Lett. 80, 1357 (1998).

[30] S. Büchner and A. Heuer, Phys. Rev. E 60, 6507 (1999).

[31] Interestingly, the "basin-sampling" approach introduced in Ref. [20] performs the reverse of the current procedure. Taking advantage of the enhanced sampling on the transformed landscape $[19,29]$ and using an approximation for the basin area, basin sampling inverts $p_{\min }^{\text {trans }}(E, T)$ to obtain $\Omega_{\min }(E)$, and hence the thermodynamic properties of the system. It is particularly useful for systems for which ergodicity is hard to achieve on the untransformed landscape.

[32] S. Sastry, P. G. Debenedetti, and F. H. Stillinger, Nature (London) 393, 554 (1998).

[33] M. Dzugutov, S. I. Simdyankin, and F. H. M. Zetterling, Phys. Rev. Lett. 89, 195701 (2002).

[34] G. T. Barkema and N. Mousseau, Phys. Rev. Lett. 81, 1865 (1998).

[35] W. Kob and H. C. Andersen, Phys. Rev. E 51, 4626 (1995).

[36] M. Dzugutov and U. Dahlborg, J. Non-Cryst. Solids 131-133, 62 (1991).

[37] F. H. Stillinger and T. A. Weber, Phys. Rev. B 31, 5262 (1985).

[38] If the probability distribution scales as $A^{-2}$ then the cumulative distribution should scale as $A^{-1}$.

[39] F. Rao and A. Caflisch, J. Mol. Biol. 342, 299 (2004). 